\title{
CADENA ESPÁRRAGO: PRODUCCIÓN DE DIFERENTES GENOTIPOS EN SU QUINTO AÑO Y COMPORTAMIENTO EN POSCOSECHA ${ }^{1}$
}

\author{
Erika Kirschenbilder ${ }^{2}$, Ana María Castagnino ${ }^{2,3}$, Karina Elizabeth Díaz ${ }^{3}$, María Belén Rosini ${ }^{3}$, \\ Agostino Falavigna 4
}

RESUMEN

Cadena espárrago: producción de diferentes genotipos en su quinto año y comportamiento en poscosecha. El objetivo del presente trabajo fue evaluar el rendimiento de primicia de cosecha en invernadero, de tres genotipos de espárrago, en su quinta temporada. El ensayo se inició en la Facultad de Ciencias Agrarias, UCA, en Buenos Aires, el 15 de noviembre de 2006. En la presente evaluación se efectuaron diecinueve cosechas manuales, del 4 de setiembre de 2012 al 22 de octubre de 2012, cada dos días. Se realizó un análisis multifactorial ANOVA-LSD test $(\mathrm{p}>0,05)$. Se evaluó la productividad total y comercial (PFT y PFC), número de turiones totales y comerciales/ha (NTT y NTC), distribución de calibres (DC): jumbo (J), extralarge (XL), large (L), medium (M) y small (S); y defectos encontrados (D): espigados (TE), cortos (TC) y otros defectos (OD). En poscosecha se evaluaron: atados (A), bandejas (BA) y bolsas de polietileno (BO), para determinar la evolución del peso fresco (EPF), pérdida diaria de peso fresco (PPFD) y peso fresco promedio del período de poscosecha (PPFP). Los genotipos italianos superaron las 9 t/ha, valor que duplica la media nacional argentina superando a UC-157. En producción, se destacó Giove en PFT, PFC, NTT, NTC y una DC favorable, con un $70 \%$ de los turiones L y XL. Con respecto al comportamiento en poscosecha, se evidenció que el menor grado de deshidratación se obtuvo mediante el empleo de bolsas y bandejas en el envasado.

Palabras clave: Asparagus officinalis var. altilis, calidad de espárragos, turiones.

\begin{abstract}
Asparagus chain: Fifth year production of different genotypes and postharvest behaviour. The objective of this investigation was to study greenhouse precocious spears yield of three genotypes of asparagus on its fifth harvest season. The trial was established at the Faculty of Agricultural, Pontifical Catholic University of Argentina, on November, 15, 2006. 19 different harvests were made from 04/09/2012 to $22 / 10 / 2012$, one every two days. A multifactor ANOVALSD test $(\mathrm{P}>0.05)$ was performed. Variables analyzed were: total productivity and commercial productivity (PFT and PFC), total number of spears and commercial number of spears (NTT and NTC); caliber distribution (DC): Jumbo (J), Extra-Large (XL), Large (L), Medium (M) and Small (S) and finally, quality defects (D): spiked spears (TE); short spears (TC) and other defects (OD). During the post-harvest period, bundles (A), trays (BA) and polyethylene bags were used to study fresh weight evolution (EPF); daily fresh weight loss (PPFD) and average fresh weight during the post-harvest period (PPFP). Italian breeds yielded above $9 \mathrm{t} / \mathrm{ha}$, twice the Argentinean national average. According to the production results, Giove stood out regarding PFT, PFC, NTT, NTC and a proper DC, where the $70 \%$ of spears measured L and XL. With regard to postharvest behavior, it was found that the lowest degree of dehydration was obtained by using bags and trays.
\end{abstract}

Keywords: Asparagus officinalis var. altilis, asparagus quality, spears.

\footnotetext{
Recibido: 29 julio, 2014. Aceptado: 4 de setiembre, 2014. Este trabajo forma parte de la tesis de grado en Ingeniería en Producción Agropecuaria del primer autor, Pontificia Universidad Católica Argentina, Buenos Aires.

2 Pontificia Universidad Católica Argentina (UCA), Facultad de Ciencias Agrarias, Cátedra de Horticultura, Freire 183, (CI426AVC) Bs. As., Argentina.erika89k@hotmail.com

3 Universidad Nacional del Centro de la Provincia de Buenos Aires (UNCPBA), Facultad de Agronomía, Centro Regional de Estudio Sistémico de Cadenas Agroalimentarias (CRESCA), República de Italia 780 (7399) Azul, Argentina. amc@ faa.unicen.edu.ar,kardiaz70@hotmail.com, belenrosini@yahoo.com.ar

4 Consiglio per la Ricerca e la Sperimentazione in Agricoltura-UnitA di Ricerca per l'Orticoltura (CRA-ORL). Montanaso Lombardo, Lodi, Italia. agostino.falavigna@entecra.it
} 


\section{INTRODUCCIÓN}

El cultivo de espárrago, Asparagus officinalis var. altilis es una hortaliza altamente perecedera donde el empleo de envases adecuados es un aspecto importante para prolongar su período de poscosecha. Actualmente se emplean distintas técnicas de envasado como bandejas y bolsas para sellado al vacío, además de los tradicionales atados. Es una hortaliza rica en componentes nutracéuticos, sustancias químicas que se encuentran como componentes naturales de los alimentos, que benefician a la salud, ya que previenen enfermedades o mejoran el estado fisiológico del individuo (Fuentes-Alventosa, 2009).

La producción a nivel mundial de este cultivo está liderada por China, con un $88,8 \%$ de la producción total de 8274335 t/año, seguido por Perú, México y Alemania, con un 4,5; 1,4 y 1,2\% respectivamente; mientras que Argentina ocupa el puesto número 15 (FAO, 2012). Fue la hortaliza a nivel global que experimentó el mayor crecimiento en producción (en porcentaje anual) durante el periodo 2000-2005 (7,8\%) (Ferratto y Mondino, 2008; Ferratto y Rodríguez, 2010).

En Argentina, la superficie y producción se expandió hasta la década del noventa, cuando llegó a 3200 ha y un volumen total producido de $8500 \mathrm{t}$ (1992), con valores de exportación que llegaron a 1700 t (1997) (Santos, 2011) y luego disminuyó a menos de 1000 ha. Actualmente existe una demanda interna creciente e insatisfecha (Pascualetti et al., 2013). El área cultivada promedio, en el período 2003 - 2012, según FAO fue de 2098 ha (FAOSTAT, 2012; Barreto, 2013). Hay un déficit del 30\%, que se cubre con importaciones de países como Perú (principal exportador mundial) (Sastre-Vázquez et al., 2010). Esta situación muestra que existen grandes oportunidades para el reposicionamiento del cultivo, tanto para el mercado interno como para la exportación en fresco (Sastre-Vázquez et al., 2010).

Los espárragos, históricamente han presentado una elevada estacionalidad en su comercialización, en correspondencia con el periodo de producción, no existiendo referencias de producción en invernadero a nivel nacional (Risso et al., 2012). Su época normal de producción es la primavera, superado el riesgo de heladas tardías, lo que normalmente ocurre en la provincia de Buenos Aires, Argentina, a partir de mediados de septiembre, fecha que puede adelantarse mediante el empleo de técnicas de protección. Entre las posibilidades actuales de extender el calendario de oferta se encuentra el empleo de invernaderos, que permiten anticipar en un mes la entrada en producción, propiciando un mejor posicionamiento en el mercado de las empresas productoras (Barreto, 2013).

En el caso de la ciudad de Buenos Aires, la temperatura promedio para el período de producción de espárragos es de $14,6{ }^{\circ} \mathrm{C}$, la máxima promedio de $29,35^{\circ} \mathrm{C}$ y la mínima de $10,6{ }^{\circ} \mathrm{C}$, según el Servicio Meteorológico Nacional (SMN, 2014). El empleo de invernaderos permite disminuir la amplitud térmica diaria y elevar las temperaturas promedio entre 3 y $5{ }^{\circ} \mathrm{C}$ según si el día está nublado o soleado (Pita et al., 1998; Kawashina y Nonaka, 2000), aspecto que propicia la producción anticipada de hortalizas como los espárragos.

Respecto del ciclo vital de las plantas de espárrago verde, este se divide en cuatro fases: de crecimiento temprano, los primeros dos años desde la plantación, caracterizados por un fuerte desarrollo vegetativo; de productividad creciente $\left(3^{\circ}-4^{\circ}\right.$ año) que corresponde a los dos primeros años de cosecha; de productividad estable $\left(4^{\circ}-10^{\circ}\right.$ año) y finalmente la de productividad decreciente (diez años en adelante). De estas, la fase correspondiente a la plantación del cultivo es la más crítica (Falavigna y Palumbo, 2001). Eventuales errores pueden afectar la producción y la calidad de los turiones cosechados, a lo largo de toda la vida útil; por esto resulta fundamental contar con una adecuada planificación del cultivo, que incluye la elección del híbrido a cultivar, el sistema de inicio, el marco de plantación, etc. La etapa de poscosecha es también muy importante, ya que a ella corresponde la posibilidad de brindar el producto a los consumidores en sus mejores condiciones (Falavigna, 2004).

La cadena agroalimentaria del espárrago presenta dos etapas principales: productiva y de poscosecha (Risso et al., 2012).

En dichas etapas, el espigado representa el principal motivo de descarte durante el procesado y de rechazos en el proceso de comercialización de turiones verdes. En este sentido, Uragami et al. (1993) señalan que el promedio de los índices de cerrado de la cabeza de los turiones comerciales que evaluaron estuvo altamente correlacionado con los mismos índices de los cuatro años de cosecha que consideraron; es decir, la condición de cerrado de la cabeza se mantuvo para cada genotipo. Dicho autor sostuvo que es posible estimar los rendimientos, el peso promedio de los 
turiones (PPT) y el cerrado de la cabeza de un cultivar, a partir de los resultados de la segunda cosecha, para un período de cuatro semanas de cosecha (Krarup y Contreras, 2002).

El espárrago es un cultivo cuyas plantas son dioicas, siendo más productivas las plantas masculinas que las femeninas (Castagnino et al., 2009). Las plantas estaminadas (masculinas) presentan mayor número de turiones mientras que las pistiladas (femeninas) tienen mayores diámetros, según Cattivelo (2002) y Risso et al. (2012). Por tal motivo, en los genotipos enteramente masculinos se observa mayor concentración de calibres.

A lo largo del mejoramiento de esta especie, se han desarrollado diferentes tipos de materiales para aumentar el rendimiento y la uniformidad del cultivo. Estos incluyen cultivares mejorados por selección masal, distintos tipos de híbridos simples, dobles, clonales y materiales constituidos por las F2 de híbridos clonales (Bannerot et al., 1969; Bussell et al., 1987). Además existen actualmente en el mercado genotipos con distinto potencial de rendimiento $\mathrm{y}$ distribución de calibres, cuyo comportamiento en campo y en invernadero, como en poscosecha, debe ser estudiado. Entre ellos se destacan los genotipos enteramente masculinos, de producción mucho más uniforme respecto de la distribución de calibres.

En cuanto a los calibres de los turiones producidos con los distintos genotipos, los consumidores en los países de Europa prefieren turiones de mayor diámetro $(\mathrm{L}, \mathrm{XL}$ y $\mathrm{J})$ y en Estados Unidos turiones de menor calibre ( $\mathrm{S}$ y $\mathrm{M}$ ), además, las exigencias en calidad son, en general, cada vez mayores. Por este motivo, resulta necesario estudiar como complemento de la productividad, la distribución de calibres, no solo en los primeros años del cultivo, donde se obtiene un crecimiento exponencial, sino fundamentalmente en la etapa adulta (Barreto, 2013) donde las plantas que dan origen a los turiones presentan yemas más desarrolladas.

Los destinos principales de esta hortaliza son el mercado en fresco de I Gama, así como conservas de II Gama y surgelados o supercongelados de III Gama (Castagnino, 2004).

Según Cowan et al. (2001), los alimentos IV Gamma o fresh-cut así como también las hortalizas frescas envasadas como los espárragos, constituyen innovaciones en la industria alimentaria por su elevado contenido de servicio y la posibilidad de mantener las características organolépticas y sensoriales de los productos frescos, permitiendo obtener un producto listo para consumir y/o fácil de usar.

Las hortalizas perecederas como los espárragos, una vez lavadas, procesadas y desinfectadas, son envasadas en atados, bolsas o en bandejas cubiertas con películas plásticas. Esto, a pesar de las dificultades inherentes a su manejo, trae consigo muchas ventajas, como por ejemplo, requerir un menor tiempo de preparación de las comidas y presentar una calidad uniforme y constante de productos frescos, saludables, ricos en nutrientes y con facilidad de almacenamiento por estar fraccionados (Viña y Chaves, 2003). Estos combinan aspectos de facilidad de uso, con inocuidad y diferenciación, representando una verdadera respuesta a los nuevos comportamientos de consumo de alimentos. Surgieron por primera vez en Estados Unidos en los años setenta donde representan un consumo real (Stampaccia et al., 2008).

En cuanto a los tipos de envase para el acondicionamiento de espárrago, según algunos autores, como Ospina-Meneses y CartagenaValenzuela (2012), pueden utilizarse para el acondicionamiento de espárrago bolsas de polietileno de baja densidad, que junto con el cloruro de vinilo, es uno de los principales plásticos utilizados en el envasado de frutas y hortalizas. En cuanto a sus ventajas se pueden citar una baja permeabilidad al vapor de agua, alta a los gases, aromas y grasas, excelente sellabilidad, bajo costo comparativo con otros materiales de empaque, claridad y moderada resistencia a la tensión, menor peso por unidad de empaque, seguridad para el consumidor final, lo cual agrega fácilmente valor al producto. Esta película es la lámina base termoformable más ampliamente utilizada para envasado en atmósfera modificada. El PVC posee una buena capacidad barrera frente a los gases y moderada al vapor de agua; además, posee una excelente resistencia a grasas y aceites (OspinaMeneses y Cartagena-Valenzuela, 2012).

A fin de propiciar un adecuado procesado de esta hortaliza en Argentina, la SAGPyA (2007) elaboró un Protocolo de Procesado en Fresco de Espárrago Verde.

En este contexto, el objetivo del presente trabajo fue evaluar el rendimiento de primicia de cosecha en invernadero, de tres genotipos masculinos italianos de espárrago, en su quinta temporada de cosecha. 


\section{MATERIALES Y MÉTODOS}

\section{Parte 1: Evaluación de la productividad de híbridos de espárrago verde}

\section{Ubicación del estudio}

El ensayo se inició el 15 de noviembre de 2006 en la Facultad de Ciencias Agrarias de la Pontificia Universidad Católica Argentina (UCA) situada en la Ciudad Autónoma de Buenos Aires, en un invernadero de techumbre curva, a través de la plantación definitiva de los plantines obtenidos en vivero.

El clima de la zona de estudio se caracteriza por ser templado húmedo (comúnmente denominado pampeano), con veranos cálidos e inviernos fríos, con precipitaciones moderadas de $1000 \mathrm{~mm}$ anuales, con mayor incidencia en la época estival, muy influido por la cercanía al Río de La Plata. Por lo general, posee una amplitud térmica diaria moderada y una variación térmica bien diferenciada entre una estación y otra. Las precipitaciones suelen ser moderadas. La temperatura media es de $16,6^{\circ} \mathrm{C}$ y humedad relativa promedio es del $71,4 \%$.

\section{Diseño de la plantación}

La plantación se inició mediante plantines de 100 días, producidos con el empleo de una mezcla de $70 \%$ de turba y $30 \%$ de perlita y vermiculita para el tapado. La densidad utilizada fue de 33333 pl/ha, y un marco de plantación de $1 \mathrm{~m} * 0,3 \mathrm{~m}$. Se emplearon tres híbridos masculinos de origen italiano y un testigo de origen americano; se contó con dos bloques de los distintos híbridos, dentro y fuera del invernadero. La profundidad de siembra fue de $25 \mathrm{~cm}$. El tamaño de cada una de las parcelas fue de $5 \mathrm{~m}^{2}$, con una cantidad de 13 plantas evaluadas por parcela.

Al momento de la plantación, se efectuó fertilización de fondo en banda con fosfato de amonio a razón de $200 \mathrm{~kg} / \mathrm{ha}$. Se realizó control químico de malezas en preemergencia con Linurón $\left(\mathrm{C}_{9} \mathrm{H}_{10} \mathrm{C}_{12} \mathrm{~N}_{2} \mathrm{O}_{2}\right)$, a razón de 2,0 l/ha. Se complementó con labores mecánicas con motocultivador entre las hileras, y manuales en la hilera durante el periodo vegetativo. Se efectuó riego por surco.

En el año de estudio el sistema de riego utilizado fue por goteo y el control de malezas efectuado fue manual y químico mediante el empleo de Metribuzim $\left(\mathrm{C}_{8} \mathrm{H}_{14} \mathrm{~N}_{4} \mathrm{OS}\right)$ y Pendimetalín $\left(\mathrm{C}_{13} \mathrm{H}_{19} \mathrm{~N}_{3} \mathrm{O}_{4}\right)$ a razón de 2 1/ha.

\section{Material genético evaluado}

En el presente trabajo se analizó la productividad total obtenida con el híbrido UC-157 de origen americano y los introducidos de origen italiano: Marte, H 668 y Giove, cuyas características fenotípicas fueron descritas por Falavigna (2006) y Risso (2012) (Cuadro 1).

Cuadro 1. Características fenotípicas de los híbridos Marte, H 668, Giove y UC-157. Buenos Aires, Argentina. 2012.

\begin{tabular}{|c|c|c|c|}
\hline Híbrido & Origen & Plantas & Características \\
\hline Marte & Italiano & $\begin{array}{c}100 \% \\
\text { masculinas }\end{array}$ & $\begin{array}{c}\text { Resistente a roya y } \\
\text { tolerante a Fusarium } \\
\text { De elevada uniformidad } \\
\text { fenotípica } \\
\text { De productividad media } \\
\text { Precoz } \\
\text { Apto para la producción } \\
\text { como verde o como } \\
\text { blanco } \\
\text { De sabor delicado y muy } \\
\text { poca fibrosidad }\end{array}$ \\
\hline Н 668 & Italiano & $\begin{array}{c}100 \% \\
\text { masculinas }\end{array}$ & $\begin{array}{l}\text { Experimental al momento } \\
\text { de iniciar el ensayo }\end{array}$ \\
\hline Giove & Italiano & $\begin{array}{c}100 \% \\
\text { masculinas }\end{array}$ & $\begin{array}{c}\text { Caracterizado por la } \\
\text { producción de turiones de } \\
\text { elevado calibre }\end{array}$ \\
\hline UC-157 & Americano & $\begin{array}{c}50 \% \\
\text { femeninas } \\
-50 \% \\
\text { masculinas }\end{array}$ & $\begin{array}{l}\text { Muy precoz } \\
\text { Brácteas cerradas aún ante } \\
\text { elevadas temperaturas } \\
\text { De mediano a bajo calibre }\end{array}$ \\
\hline
\end{tabular}

\section{Cosecha de turiones}

El período de cosecha fue el 04/09/2012 hasta el 22/10/2012, periodo durante el cual se realizaron diecinueve recolecciones, cada dos o tres días, tal como lo sugiere Farías et al. (2004).

Los turiones se cosecharon una vez que alcanzaron los 23 - $24 \mathrm{~cm}$ siguiendo las indicaciones del protocolo de calidad de espárrago fresco para Argentina (SAGPyA, 2007) mencionado y se trasladaron al laboratorio para su procesamiento, el cual consistió 
en lavado, pesado, selección por calidad, por calibre, corte y acondicionamiento.

\section{Análisis estadístico}

En este ensayo se efectuó un análisis multifactor ANOVA-LSD test $(\mathrm{P}>0,05)$, mediante el cual se estudiaron las variables: diferencias en productividad total y comercial (PFT y PFC), número de turiones producidos (NT) y distribución porcentual de calibres (DC) y defectos encontrados (D): espigados (TE); cortos (TC), y otros defectos (OD).

La distribución de calibres considerada se realizó en función del diámetro a $2,5 \mathrm{~cm}$ de la base de los turiones: jumbo (J): con más de $18 \mathrm{~mm}$, extra-large (XL): de 16 a $18 \mathrm{~mm}$, large (L): de 16 a $12 \mathrm{~mm}$, medium (M): de 12 a $9 \mathrm{~mm}$ y small (S): de 9 a $6 \mathrm{~mm}$, tomando como referencia los valores indicados en el mencionado Protocolo de Calidad de Espárragos Frescos de Argentina (SAGPyA, 2007).

\section{Parte 2: Evaluación de poscosecha}

En esta etapa del ensayo, se evaluó la incidencia de tres tipos de envasado de espárrago verde: atados (A), bandejas (BA) y bolsas (BO) de $500 \mathrm{~g}$, para turiones de $22 \mathrm{~cm}$ de largo, los cuales fueron producidos en los ensayos de genotipos efectuados en la Facultad de Ciencias Agrarias de la Pontificia Universidad Católica Argentina (UCA).

\section{Ubicación del estudio}

El período de poscosecha se extendió desde el 17/10/2012 al 07/11/2012. Tanto las tareas de acondicionamiento para IV Gama como el período de almacenamiento con temperaturas menores de $4{ }^{\circ} \mathrm{C}$, fueron llevados a cabo en un laboratorio de la Facultad de Ciencias Agrarias, de la Pontificia Universidad Católica Argentina (UCA), en la ciudad de Buenos Aires.

\section{Distintos envases evaluados}

Atados (A): es la forma más usual que utilizan los productores de espárragos en el país, la misma consta de un conjunto de turiones unidos por un hilo o una bandita elástica, sin ninguna protección o aislación con el medio, lo cual puede dar lugar a contaminaciones del alimento y a un mayor grado de deshidratación.

Bolsas de polietileno de baja densidad (BO): en este caso, los turiones, una vez acondicionados, se almacenaron dentro de bolsas transparentes y cerradas herméticamente.

Bandejas de poliestireno expandido (BA): los turiones se presentaron en dichas bandejas recubiertos de film de PVC transparente (Resinite AF 50), apto para alimentos de alta densidad $\left(1,36 \mathrm{~g} / \mathrm{cm}^{3}\right)$.

\section{Secuencia de procesado en fresco}

La secuencia utilizada para el procesado de los turiones cosechados fue la siguiente: lavado, selección, corte a $22 \mathrm{~cm}$, calibrado y envasado. En esta etapa también se siguieron las recomendaciones del Protocolo de Calidad para Espárrago Verde de Argentina (SAGPyA, 2007), y una vez acondicionados fueron almacenados a $4{ }^{\circ} \mathrm{C}$.

\section{Variables en estudio}

La evaluación de comportamiento en poscosecha se llevo a cabo durante un período de veinte días (17/10/2012-07/11/2012), en el cual se efectuaron seis determinaciones, con tres repeticiones en cada caso, de las siguientes variables:

- Evolución del peso fresco (EPF).

- Pérdida de peso fresco diaria (PPFD).

- Peso fresco promedio del período de poscosecha (PPFP).

\section{Análisis estadístico}

En esta segunda etapa, también se efectuó un análisis multifactorial ANOVA prueba de comparación de medias LSD $(\mathrm{p}>0,05)$ para todas las variables analizadas.

\section{RESULTADOS Y DISCUSIÓN}

Se adelantó el periodo de cosecha en diecisiete días, respecto de otros cultivos de híbridos de espárrago verde en la Provincia de Buenos Aires, lo que permite 
anticipar el posicionamiento en el mercado, propiciando la extensión del período de oferta.

\section{Parte 1: Productividad de híbridos de espárrago verde en invernadero}

\section{Producción total lograda}

Para la variable productividad fresca total (PFT) se obtuvieron en promedio: 9,9 t/ha, encontrándose diferencias significativas para la variable híbridos. Se destacaron los genotipos masculinos italianos según el siguiente orden de importancia: Giove (a), seguido por Marte y H-668 (ab) tal como se observa en el Cuadro 2.

Cuadro 2. Productividad (número de turiones comerciables NTC y productividad total de turiones PFT) en invernadero de cuatro híbridos de espárrago verde en su quinto período productivo. Buenos Aires, Argentina. 2012.

\begin{tabular}{lcc}
\hline Híbrido & $\begin{array}{c}\text { Peso fresco total } \\
\text { (kg/ha) }\end{array}$ & $\begin{array}{c}\text { Número total de } \\
\text { turiones cosechados } \\
\text { turiones/ha }\end{array}$ \\
\hline UC-157 & $7355 \mathrm{~b}$ & $342500 \mathrm{~b}$ \\
Marte & $9268 \mathrm{ab}$ & $440000 \mathrm{~b}$ \\
H 668 & $9705 \mathrm{ab}$ & $814999 \mathrm{a}$ \\
Giove & $13619 \mathrm{a}$ & $692501 \mathrm{a}$ \\
\hline
\end{tabular}

Letras distintas en superíndices indican diferencias significativas según la prueba de LSD $(\mathrm{p}<0,05)$.

En cuanto a la variable productividad fresca comercial (PFC) también se destacó Giove (a), seguido de UC-157 y H-668 (ab) y finalmente Marte (b) (Cuadro 2).

La productividad por planta fue en promedio de $300 \mathrm{~g}$, destacándose el híbrido Giove con $409 \mathrm{~g} /$ planta, seguido de H668 con 291 g/planta y Marte con $278 \mathrm{~g} /$ planta y, finalmente UC-157 con $221 \mathrm{~g} /$ planta. Dichos resultados muestran la gran evolución en productividad respecto del primer año evaluado (2008) en que se destacara UC-157 con $251 \mathrm{~g} /$ planta seguido de Giove con $184 \mathrm{~g} /$ planta, H668 con 135 y finalmente Marte con $45 \mathrm{~g} /$ planta. El único híbrido que redujo el rendimiento en los cinco años de estudio (un 12\%) fue UC-157, mientras todos los demás aumentaron: $618 \%$ Marte seguido de Giove con 222\% y H-668 con
$215 \%$. Esto implica que, en el caso de cultivar UC 157 en invernadero debe tenerse en cuenta que presenta un corto ciclo de vida en plena producción y que a los pocos años esta puede comenzar a disminuir.

Similar tendencia se observó en la cosecha 2011 con los genotipos italianos, según Risso et al. (2012), quien indicó que Giove logró una productividad de 9062 (a) kg/ha, H-668 de 6187 kg/ha (ab) y Marte de 3625 (b). Diferente comportamiento mostró el híbrido UC-157, ya que en dicho año (correspondiente a la cuarta temporada de evaluación) tuvo un rendimiento de 8437 (a) kg/ha, lo que estaría mostrando que el híbrido UC-157 se encuentra en la etapa de productividad decreciente.

El presente ensayo en su primera evaluación productiva, realizada en el año 2008, rindió un promedio de $5133 \mathrm{~kg} / \mathrm{ha}$, destacándose en orden de importancia: UC-157 con 8375 (a) kg/ha, seguido de Giove con 6147 (b) $\mathrm{kg} / \mathrm{ha}, \mathrm{H}-668$ con 4510 (b) $\mathrm{kg} / \mathrm{ha}$ y Marte con (c) $1500 \mathrm{~kg} / \mathrm{ha}$ (Castagnino, 2009). Estos resultados indican la conveniencia de cultivar UC-157 cuando el objetivo es lograr elevada productividad en el primer año y que, de los tres genotipos masculinos, Giove es el que mostró su superioridad productiva desde el primer año.

Respecto a la producción comercial de turiones de primera calidad a lo largo del presente período de cosecha, la misma varió entre $226 \mathrm{~kg} /$ cosecha y 1018 $\mathrm{kg} /$ cosecha. Se destacaron las cosechas: $\mathrm{N}^{\circ} 16$ con 1018 (a), seguida de la $\mathrm{N}^{\circ} 1$ con 965 (ab), la $\mathrm{N}^{\circ} 19$ con 899 (abc), la $\mathrm{N}^{\circ} 11$ con $845 \mathrm{~kg} /$ cosecha (abcd) tal como se observa en la Figura 1. Dichas variaciones

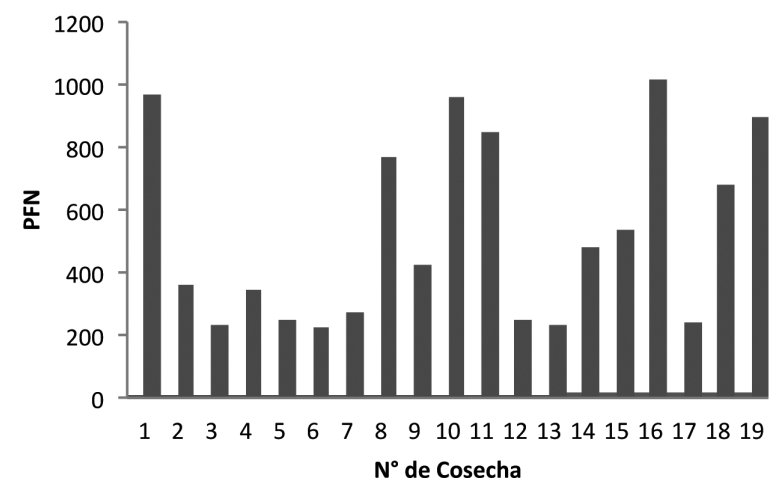

Figura 1. Evolución de la productividad fresca neta (PFN) comercial de primera calidad de cuatro híbridos de espárrago verde a lo largo del período de cosecha del 4 setiembre al 22 de octubre del 2012. Buenos Aires, Argentina. 2012. 
resultan normales ya que se trata de una técnica de protección de semiforzado, es decir, de invernadero frío, sujeto a las normales variaciones de temperatura de fin de invierno y comienzo de primavera que caracterizan la zona de estudio.

El número promedio total de turiones cosechados fue 572500 , encontrándose diferencia estadística significativa para los híbridos en estudio. Se destacaron Giove (a) y H-668 (a), seguido de Marte (b) y UC-157 (b) (Cuadro 2), los que produjeron un promedio de 24, 21, 13 y 10 turiones/planta, respectivamente. Estos resultados difieren de los obtenidos en el primer año evaluado (2008) del presente ensayo (Castagnino et al., 2012), cuyo promedio general fue de doce turiones/planta y se destacaron los genotipos Marte con 21 turiones/ planta, UC-157 con 14 y finalmente, H-668 y Giove con un total de siete turiones/planta. Dichas diferencias indican que la curva productiva de turiones de H-668 y Giove fue más lenta durante los primeros años de la plantación, respecto de la de Marte y UC 157, ya que en el presente (quinto) período de cosecha ha manifestado una menor adaptación genotipo - ambiente, por lo que su cultivo no sería recomendable en condiciones de semiforzado. Esto estaría indicando la conveniencia de iniciar plantaciones con estos dos últimos híbridos solo cuando el objetivo es lograr elevada productividad los primeros años, pero que cuando el objetivo es lograr elevados rendimientos también en el largo plazo, convendría el cultivo de Giove y H-668. No obstante, resulta conveniente continuar evaluando las tendencias productivas de dichos genotipos, algunos años más.

En cuanto a la evolución de la producción promedio de turiones por cosecha, el mismo varió entre 12500 y más de 51000 , destacándose las cosechas 10 y 16 con 51250 y 48750 (a) turiones/ cosecha, respectivamente, seguidas de la 4 con 46250 y la 1 con 42500 (Figura 2).

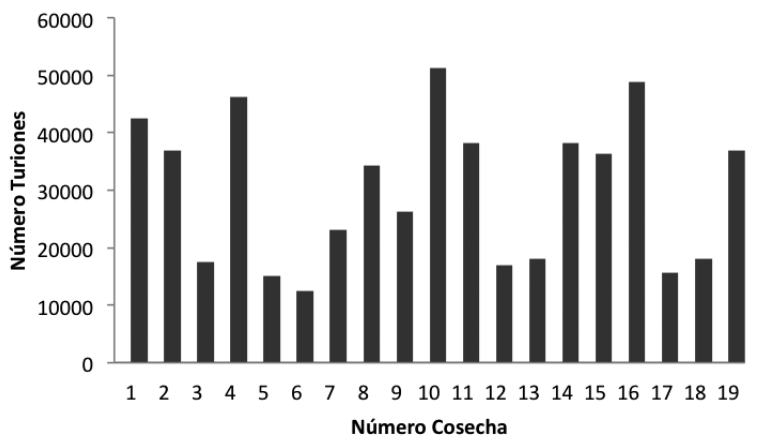

Figura 2. Productividad de turiones de cuatro híbridos de espárrago verde en invernadero, a lo largo del período de cosecha del 4 de setiembre al 22 de octubre del 2012. Buenos Aires, Argentina. 2012.

\section{Distribución de calibres}

Respecto de la distribución porcentual de calibres logrados (DC) se obtuvieron en J: 3,4\%; XL: 11,9\%; L: $42,5 \%$; M: 25,9\% y S: 16,4\%. Solo hubo diferencias para calibres L y S (Cuadro 3).

El 57,8\% de los turiones totales correspondió a los mayores calibres (J, XL y L), destacándose en orden de importancia: Giove (74\%), Marte (60\%), UC-157 $(49,9 \%)$ y H $668(47,1 \%)$. A los menores calibres (M y S) correspondió el $42,3 \%$ de la producción general, destacándose en orden de importancia H668 $(52,9 \%)$, UC $157(50 \%)$, Marte (40\%) y finalmente, Giove (26\%).

Estos resultados indican la mayor predisposición a producir turiones de elevado calibre de los genotipos Giove y H-668. Por tal motivo, convendría cultivar el híbrido Giove cuando el objetivo es el de abastecer mercados con elevadas exigencias respecto de los calibres, como es el caso de la Unión Europea.

Cuadro 3. Distribución porcentual de calibres de turiones de cuatro híbridos de espárrago verde producidos en invernadero, en su quinta temporada de evaluación. Buenos Aires, Argentina. 2012.

\begin{tabular}{lccccc}
\hline Híbrido & Jumbo (\%) & Extra-large (\%) & Large (\%) & Medium (\%) & Small (\%) \\
\hline Marte & 0 & 20,0 & 40,0 & 33,3 & 6,7 \\
UC-157 (testigo) & 3,8 & 11,5 & 34,6 & 34,6 & 15,4 \\
H668 & 5,9 & 5,9 & 35,3 & 23,5 & 29,4 \\
Giove & 4,0 & 10,0 & 60,0 & 12,0 & 14,0 \\
\hline
\end{tabular}


En el caso del genotipo UC-157, presenta el $50 \%$ de plantas femeninas, productoras de turiones de mayor calibre pero en menor cantidad y $50 \%$ de plantas masculinas, productoras de un mayor número de turiones pero de menor calibre, debido a esta causa, puede observarse en la Figura 3, una mayor dispersión de calibres.

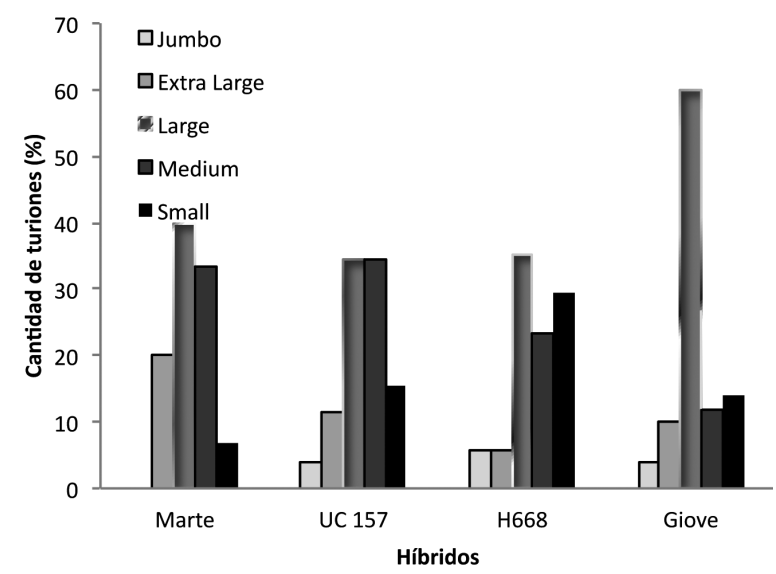

Figura 3. Distribución porcentual de calibres producidos en invernadero por cuatro híbridos de espárrago verde evaluados durante su quinta cosecha. Buenos Aires, Argentina. 2012.

\section{Defectos encontrados}

Respecto de los defectos encontrados (D) en la quinta temporada de evaluación, se destacó el defecto de espigado (TE) con un promedio de 310000 turiones/ ha, seguido de turiones cortos (TC) con $121250 \mathrm{y}$ otros defectos (OD) con 65 000, como daños de plagas y enfermedades, entre otros.

La elevada cantidad de turiones espigados obtenidos en general, indica la necesidad de bajar el largo de corte de los turiones, a fin de optimizar la proporción de turiones comerciales de primera calidad. Respecto de los TE por genotipo, se encontró este defecto principalmente en: H-668, seguido de Giove, Marte y UC-157. Esto confirma lo sostenido por algunos autores respecto de la mayor predisposición del híbrido UC-157 a mantener las brácteas cerradas, aun cuando son sometidos a las elevadas temperaturas diurnas que pueden tener los invernaderos en la zona de estudio.
TC en H-668, Giove; Marte y UC-157; mientras que OD se encontraron en orden de importancia en Giove seguido de los restantes (Cuadro 4).

Cuadro 4. Defectos encontrados en turiones de espárrago verde producidos en invernadero en su quinta cosecha. Buenos Aires, Argentina. 2012.

\begin{tabular}{lccc}
\hline Híbrido & $\begin{array}{c}\text { Cortos } \\
\text { (TC) }\end{array}$ & $\begin{array}{c}\text { Otros } \\
\text { Defectos (OD) }\end{array}$ & $\begin{array}{c}\text { Espigados } \\
\text { (TE) }\end{array}$ \\
\hline UC-157 & $60000 \mathrm{~b}$ & $32499 \mathrm{~b}$ & $177500 \mathrm{c}$ \\
Marte & $92500 \mathrm{~b}$ & $67500 \mathrm{~b}$ & $244999 \mathrm{bc}$ \\
Giove & $120000 \mathrm{ab}$ & $132500 \mathrm{a}$ & $314999 \mathrm{~b}$ \\
H668 & $212500 \mathrm{a}$ & $27500 \mathrm{~b}$ & $502501 \mathrm{a}$ \\
\hline
\end{tabular}

Letras distintas en superíndices indican diferencias significativas según la prueba de LSD $(\mathrm{p}<0,05)$.

La predominancia del defecto de espigado estaría indicando la necesidad de disminuir el largo de corte en los turiones en cosecha.

La elevada predominancia del defecto de espigado (Figura 4), indica la necesidad de incrementar la frecuencia de cosecha, efectuando recolecciones diarias o dos veces al día, a fin de optimizar la calidad y cantidad de turiones cosechados, ya que dos tercios de los turiones defectuosos encontrados se debió a este defecto.

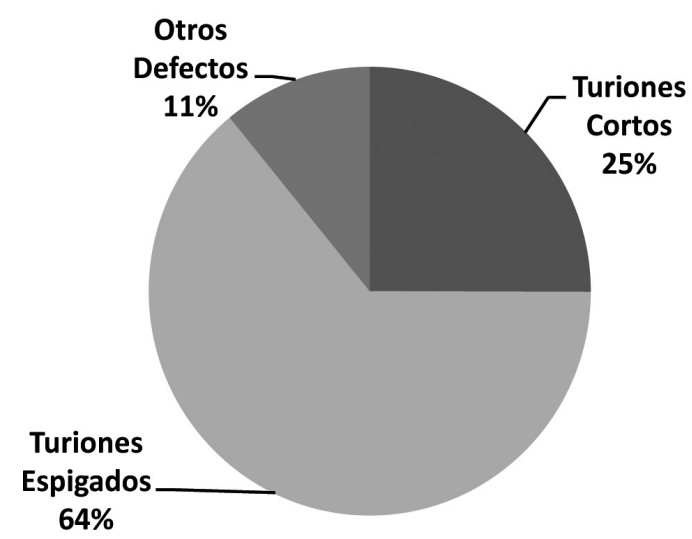

Figura 4. Distribución porcentual de defectos encontrados en la producción de turiones correspondientes a cuatro híbridos de espárrago verde, en invernadero. Buenos Aires, Argentina. 2012. 


\section{Parte 2: Comportamientoen poscosechade espárrago verde en distintas formas de acondicionado}

En la evaluación realizada, el mejor comportamiento se logró con los espárragos envasados en bandejas, las que permitieron extender el periodo de cosecha a veintiún días, con una pérdida de peso fresco del $1 \%$.

Los turiones almacenados mediante los tres tipos de envases utilizados, mantuvieron su calidad comercial durante el período comparativo de estudio y solo en la presentación de atados, se observó deshidratación en la base de estos.

El peso fresco correspondiente a las presentaciones en bolsas y bandejas no presentó diferencias estadísticas significativas entre ambas. El peso promedio durante el período comparativo de veinte días, fue de 499,78 g para las bolsas (BO) y de 493,72 g para las bandejas (BA); mientras que sí se pudo apreciar diferencia significativa entre estas y los atados (A), cuyo peso promedio comparativo para el mismo período fue de $479 \mathrm{~g}$.

Respecto de la evolución general del peso fresco (EPF) durante el periodo de estudio, el mismo no mostró diferencias significativas durante las primeras cinco determinaciones, mientras que si se pudo apreciar una diferencia significativa entre estas y la última determinación, donde el peso fresco (PF) bajó a $476 \mathrm{~g}$ en promedio, correspondiente a 95,2\% del peso total inicial por unidad. El 4,8\% de pérdida en el período de evaluación corresponde a un $1 \%$ cada cuatro días, tal como se observa en la Figura 5.

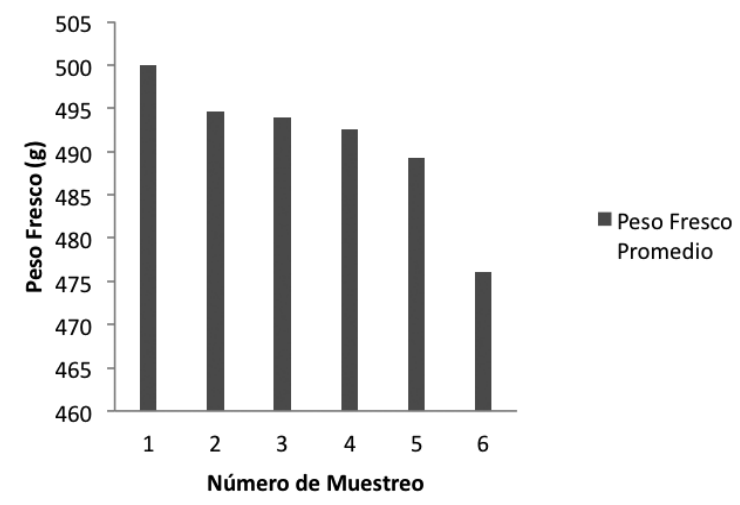

Figura 5. Evolución del peso fresco promedio de turiones de cuatro híbridos de espárrago verde a lo largo del período de poscosecha (4 setiembre al 22 de octubre del 2012). Buenos Aires, Argentina. 2012.
En el caso del empleo de bolsas, estas permitieron conservar casi el $100 \%$ del peso fresco, aunque por un período levemente menor a las bandejas, de diecisiete días. El peor comportamiento se logró con los atados cuya calidad comercial se prolongó por diecinueve días.

Respecto de las tres presentaciones, la duración del período de poscosecha evaluado para los atados sin film y para las bandejas fue de dieciséis días. En el caso de las bolsas la duración fue de diecisiete días.

En cuanto a la pérdida de peso de cada presentación se observó en atados, un peso final promedio de 456,5 g, con una pérdida de peso del 8,7\%; para las bandejas, un peso final de $498 \mathrm{~g}$, con una pérdida de peso del $0,4 \%$ y por último, para las bolsas, el peso final promedio fue de $499,8 \mathrm{~g}$, sin observarse prácticamente pérdida de peso $(0,04 \%)$ (Cuadro 5).

Cuadro 5. Peso fresco final en gramos y pérdida de peso en porcentaje (\%) y gramos (g) para las distintas presentaciones de turiones largos de espárrago verde luego de un período de almacenamiento de diecisiete días a $4{ }^{\circ} \mathrm{C}$. Buenos Aires, Argentina. 2012.

\begin{tabular}{lccc}
\hline Envase & $\begin{array}{c}\text { Peso fresco } \\
\text { final }(\mathbf{g})\end{array}$ & $\begin{array}{c}\text { Pérdida de } \\
\text { peso }(\mathbf{g})\end{array}$ & $\begin{array}{c}\text { Pérdida de } \\
\text { peso }(\boldsymbol{\%})\end{array}$ \\
\hline Atados & $456,5 \mathrm{~b}$ & 43,5 & 8,7 \\
Bandejas & $498,1 \mathrm{a}$ & 1,9 & 0,38 \\
Bolsas & 199,8 a & 0,2 & 0,04 \\
\hline
\end{tabular}

Letras distintas en superíndices indican diferencias significativas según la prueba de LSD $(\mathrm{p}<0,05)$.

En el caso de la presentación en atados, la pérdida de peso fresco resultó inferior a la indicada por Barreto (2013), quien en un estudio de trece días, tuvo una pérdida superior al $30 \%$, posiblemente debido a diferentes condiciones de almacenamiento.

La significativa diferencia entre el empleo de atados respecto de las bolsas y bandejas encontrada (Figura 6), se corresponde con la indicada por Guisolis et al. (2010), quienes señalaron la conveniencia del empleo de film para el envasado de espárrago verde, a fin de propiciar la máxima extensión posible del periodo de almacenamiento y comercialización; dichos autores encontraron que la diferencia promedio general de deshidratación de espárragos, durante el periodo de evaluación de poscosecha fue un $26 \%$ menor mediante el empleo de film. No existe información publicada 


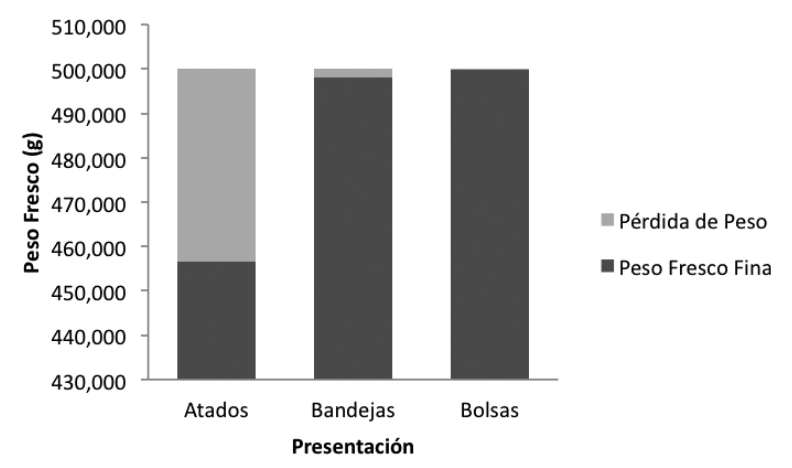

Figura 6. Comportamiento de turiones largos de cuatro híbridos de espárrago verde ante el almacenamiento por diecisiete días a $4^{\circ} \mathrm{C}$ según los diferentes envases utilizados. Buenos Aires, Argentina. 2012.

respecto al empleo de bolsas para el almacenamiento de espárrago verde.

Los resultados logrados respecto del empleo de bandejas, en relación con los atados, durante la etapa de poscosecha, coinciden con los obtenidos por Barreto (2013) quien indicó que el empleo de bandejas (B) con film permitiría extender la vida útil de los turiones de espárragos verdes frescos, ya que se conservarían por más tiempo sin alterar significativamente su peso inicial, respecto del uso de atados (A) convencionales sin film; pudiéndose comprobar que, mediante el uso de $\mathrm{B}$, la pérdida de peso fresco en el periodo de referencia fue progresivo, no así con A.

El período de duración del presente ensayo fue similar al informado por Nuñez y Casas (2000), quien estudiando la duración de la calidad de turiones almacenados en cámara fría, encontró que la misma fue de diecinueve días.

Estos resultados demuestran la conveniencia del empleo de envases en espárrago verde, en lugar de los tradicionales atados; ya sea, mediante la utilización de bolsas como también bandejas, para la optimización de la calidad del producto final.

\section{AGRADECIMIENTOS}

Se agradece la valiosa colaboración de la Srta. Cecilia Giménez Azara y a la Lic. Andrea Guisolis en las etapas iniciales que dieron origen al presente trabajo.

\section{LITERATURA CITADA}

Bannerot, H., M. Derieux, L. Thevenin, y J. Arnoux. 1969. Resultado de un ensayo comparativo de poblaciones de espárragos. (En francés) Annales de l'amélioration des plantes 19(3):289-324.

Barreto, M.S. 2013. Producción de primicia en invernadero de híbridos masculinos de espárrago (Asparagus officinalis) y procesado IV gama para optimización del posicionamiento en el mercado. http://bibliotecadigital. uca.edu .ar/repositorio/tesis/produccion-primiciainvernadero-hibridos.pdf (Consultado 17 jun. 2014).

Bussell, W.T., G.P. Falloon, y A.S. Nikoloff. 1987. Evaluación del comportamiento del rendimiento de espárrago luego de dos años de cosecha. (En inglés) New Zeal. J. Exp. Agric. 15:205-208.

Castagnino, AM. 2004. Planeamiento estratégico de la empresa agroindustrial. Editorial Hemisferio Sur, S. A. Buenos Aires, Argentina.

Castagnino, A.M., K.E. Díaz, y M.B. Rosini. 2009. Manual de cultivos hortícolas innovadores. Editorial Hemisferio Sur, Buenos Aires, Argentina.

Castagnino, A.M., K.E. Díaz, M. Rosini, M. Pascualetti, A. Guisolis, A. Novella, y A. Falavigna. 2012. Productividad a campo de ocho híbridos de espárrago verde (Asparagus officinalis var. Altilis L.) en su cuarto período de evaluación. En: Memorias XXXV Congreso Argentino de Horticultura. ASAHO, Corrientes, Argentina. 25 de setiembre de 2012. p. 309.

Cattivelo, C. 2002. El espárrago blanco. (En italiano) Bioagricultura 76:33-34.

Cowan, C., T. Cronin y M. Gannon. 2001. Mercado de los alimentos preparados y las actitudes del consumidor hacia los alimentos preparados. (En inglés) El consumidor de alimento a principios del siglo XXI, $71^{\circ}$. Año 2001, EAAE, España.

Falavigna, A. 2004. Strategia per ottimizzare e valorizzare la produzione di asparago in Sicilia. Programma Interregionale de Ricerca e Sperimentazione Applicata, Transferimento delle Innovazione agli operatori di Filiera e Programmi a forte contenuto innovativo. Editorial Grillo y Famá, Italia.

Falavigna, A. 2006. Los puntos críticos del espárrago a campo y en poscosecha. (En italiano) L'informatore Agrario 62(1):52-56.

Falavigna, A y A.D. Palumbo. 2001. La coltura dell' asparago. Editorial Calderini Edagricole, Bologna, Italia. 
FAOSTAT. 2012. Base de Datos. Organización de las Naciones Unidas para la Agricultura y la Alimentación. Estadísticas actualizadas el 23 de febrero de 2012. http://faostat3.fao. org/home/index_es.html?locale=es\#HOME (Consultado 21 jul. 2014).

Farías, V., C. Krarup, y S. Contreras. 2004. Efectos de población sobre rendimiento y calidad de turiones de cuatro cultivares de espárrago. Cien. Inv. Agr. 31:119-127.

Ferrato, A.J., y F. Rodríguez. 2010. Buenas prácticas agrícolas para la agricultura familiar: cadena de las principales hortalizas de hoja en Argentina. Organización Mundial de las Naciones Unidas para la Agricultutra y la Alimentación (FAO), Ministerio de Agricultura Ganadería y Pesca de la Nación Argentina, Instituto Nacional de Tecnología Agropecuaria (INTA) y Universidad Nacional de Rosario, Buenos Aires, Argentina.

Ferrato, J., y C.M. Mondino. 2008. Producción, consumo y comercialización de hortalizas en el mundo. Revista Agromensajes. p. 24. http://www.fcagr.unr.edu.ar/ Extension/Agromensajes/24/4AM24.htm (Consultado 10 jul. 2014).

Fuentes-Alventosa, J.M. 2009. Caracterización de componentes bioactivos del espárrago verde: obtención de ingredientes funcionales a partir de los subproductos generados durante su transformación industrial. http://helvia.uco.es/xmlui/bitstream/ handle/10396/2756/9788469293881.pdf?sequence=1 (Consultado 07 de jul. 2014).

Guisolis, A., A.M. Castagnino, K. Díaz, P. Sastre-Vázquez. J. Marina, y A. Zubiría. 2010. Impacto de técnicas innovadoras aplicadas a la cadena agroalimentaria espárrago (Asparagus officinalis L.) para optimizar el posicionamiento en diferentes mercados. Rev. Venez. Cienc. Tecnol. Aliment. 1:95-112.

Kawashina, H., y M. Nonaka. 2000. Características del entorno térmico en un invernadero con pendiente. (En inglés). Acta Hortic. 519:181-189.

Krarup, C., y S. Contreras. 2002. Elongación y ramificación de turiones de espárrago durante una cosecha primaveral. Agric. Téc. Chile 6:191-200.

Nuñez, E.G., y A.D. Casas. 2000. Composición nutricional de turiones de espárrago verde (Asparagus officinalis L.) y su relación con la vida post-cosecha. Tesis de Maestría. Producción Agrícola. UNALM, Lima, Perú.

Ospina-Meneses, S.M., y J.R. Cartagena-Valenzuela. 2012. La atmósfera modificada: una alternativa para la conservación de los alimentos. Rev. Lasallista Investig. 5(2):112-123.
Pascualetti, M., A.M. Castagnino, B.M. Rosini, M. Durante, M., y A. Zubiría. 2013. Margen bruto de diferentes híbridos de espárrago verde (Asparagus officinalis var. altilis L.), en la provincia de Buenos Aires. Rev. Colomb. Cienc. Hort. 7:201-216.

Pita, G. P.A., Pontes, M., y A. Vargues. 1998. Balance de energía de un invernadero mediterraneo. (En inglés). Acta Hort. (ISHS) 456:375-382. http://www.actahort. org/books/456/456_45.htm (Consultado 21 jul. 2014).

Risso, A., A.M. Castagnino, K. Díaz, B.M. Rosini, J. Marina, y A. Falavigna. 2012. Productividad y calidad de cuatro híbridos de espárrago verde (Asparagus officinalis L. var. altilis) en invernadero. Rev. Col. Cienc. Hort. 6:55-66.

SAGPyA (Secretaría de Agricultura, Ganadería, Pesca y Alimentos). 2007. Protocolo de calidad para espárrago fresco. Código: SAA010. Versión 08. http://www. alimentosargentinos.gov.ar/contenido/sello/sistema_ protocolos/SAA010_Esparrago_v08.pdf (Consultado 18 jun. 2014).

Santos, B. 2011. Análisis económico del empleo de dos híbridos (UC-157 y Italo) de espárrago verde con destino a diferentes mercados. http://bibliotecadigital. uca.edu.ar/repositorio/tesis/analisis-economicoempleo-dos-hibridos.pdf (Consultado 16 jun. 2014).

Sastre-Vázquez, P., A. Guisolis, A.M. Castagnino, K. Díaz, J. Marina. y A. Zubiría. 2010. Impacto de técnicas innovadoras aplicadas a la cadena agroalimentaria espárrago (Asparagus officinalis L.) para optimizar el posicionamiento en diferentes mercados. Rev. Venez. Cienc. Tecnol. Aliment. 1:95-112.

SMN (Servicio Meteorológico Nacional). 2014. Características climáticas de la ciudad de Buenos Aires. http://www.smn.gov.ar/serviciosclimaticos/?m od=elclima\&id=10 (Consultado 6 jul. 2014).

Stampacchia, P., M. Colurcio, y T.R. Spena. 2008. Preferencias, perfiles y tendencias en el consumo de productos de IV Gama. (En italiano). http://www.escpeap.net/conferences/marketing/2008_cp/Materiali/ Paper/It/Stampacchia_Colurcio_RussoSpena.pdf (Consultado 18 jun. 2014).

Uragami, A., M. Nagai, y H. Yoshikawa. 1993. Ensayo de evaluación temprana del rendimiento, peso de turiones y apertura de puntas de turiones en cultivares de espárrago. (En inglés). Acta Hort. 415:97-104.

Viña, S.Z., y A.R. Chaves. 2003. Tecnologías aptas para la conservación de hortalizas. http://anterior.inta.gov.ar/ ediciones/idia/horticola/hortalizas05.pdf (Consultado 18 jun. 2014). 
